\title{
Powassan Meningoencephalitis: A Case Report Highlighting Diagnosis and Management
}

\author{
Jolanta J. Pach ${ }^{1}$, Adeel S. Zubair ${ }^{1}$, Christopher Traner $^{1}$, Guido J. Falcone ${ }^{1}$, Jeffrey J. Dewey ${ }^{1}$ \\ 1. Neurology, Yale School of Medicine, New Haven, USA
}

Corresponding author: Jeffrey J. Dewey, jeffrey.dewey@yale.edu

\begin{abstract}
Powassan virus (POWV), a rare flavivirus that may be transmitted by a tick bite, causes rare but severe cases of encephalitis, meningitis, and meningoencephalitis in humans. We present the case of a 62-year-old man with prior Lyme disease and reactive arthritis who presented to the hospital with symptoms of fever, headache, and fatigue. The patient developed rapid deterioration of mental status including profound expressive aphasia and required intubation and high-dose steroids. Cerebrospinal fluid (CSF) serologies were
\end{abstract} found to be positive for the POWV.

Categories: Neurology

Keywords: powassan virus, viral meningitis, viral encephalitis, chronic lymphocytic leukemia, tick-borne flavivirus

\section{Introduction}

Infections of the Powassan virus (POWV) have been described in the Northern United States, Canada, and Russia [1]. The virus is transmitted by ixodid ticks and causes severe encephalitis and meningitis in humans. While rare, infections with POWV carry a $10-15 \%$ fatality rate, with some reports exceeding $20 \%$ [2]. Longterm sequelae of disease are also common [3, 4]. The symptoms reported most frequently in POWV case studies include fever, headache, confusion, generalized weakness, encephalopathy, neurological symptoms, focal deficit, and vomiting [5]. Common long-term sequelae include generalized weakness, cognitive difficulties, speech difficulties, imbalance and difficulty walking, spastic quadriplegia, ophthalmoplegia, and headaches [6]. Fewer than 300 cases of POWV have been reported since the virus was first discovered in 1958 , but cases have been steadily increasing in the United States over the past decade [2, 3]. Serologic testing is the gold standard for diagnosis, specifically immunoglobulin M (IgM) antibody testing utilizing enzyme-linked immunosorbent assay and immunofluorescence antibody. However, only state health departments and the CDC can perform Powassan-specific serologic testing [5]. There is currently no treatment or vaccine available, and supportive care is the mainstay of treatment. We present a case of a 62 year-old man presenting with POWV meningoencephalitis and discuss the workup of such patients, as well as the progression of imaging findings often reported with POWV, characterized by the appearance and subsequent resolution of radiologic hyperintensity on T2-weighted MRI. Little is known about conditions that might predispose patients to severe POWV and its long-term consequences. Given the patient's past medical history of chronic lymphocytic leukemia (CLL), we discuss the role of immunosuppression in

Review began 06/07/2021 Review ended 07/15/2021 Published 07/23/2021

\section{(c) Copyright 2021}

Pach et al. This is an open access article distributed under the terms of the Creative Commons Attribution License CC-BY 4.0., which permits unrestricted use, distribution, and reproduction in any medium, provided the original author and source are credited. neuroinvasive infection and advocate for a higher index of suspicion for neuroinvasive infection in immunosuppressed patients.

\section{Case Presentation}

A 62-year-old man with a past medical history significant for CLL, Lyme disease, and reactive arthritis presented to the hospital with fevers, headaches, and fatigue. At baseline, the patient was highly functioning and worked as a financial manager. He was not on any medications for his CLL. The patient's wife reported that the previous year he was diagnosed with reactive arthritis and started on methotrexate, which he discontinued 10 days prior to presentation on the advice of a holistic care provider in favor of a probiotic. In late May, he was otherwise in his normal state of health until a few days prior to presentation when he developed fatigue and night sweats. Subsequently, he developed headaches, malaise, and fever, at which point he presented to our hospital. His temperature was 102.1 Fahrenheit, pulse 94 beats per minute, blood pressure 158/84 $\mathrm{mmHg}$, and oxygen saturation 95\% on room air. Urgent cerebrospinal fluid (CSF) analysis showed 122 RBCs per microliter, 59 white blood cells per microliter (88\% lymphocytes, 3\% polymorphonuclear cells, $9 \%$ monocytes), protein of $98.9 \mathrm{mg} / \mathrm{dL}$, and a glucose of $80 \mathrm{mg} / \mathrm{dL}$ (Table 1). CSF and blood cultures were obtained. A broad panel of additional CSF and serum labs was ordered as detailed in Table 2. 


\section{Cureus}

\begin{tabular}{|c|c|c|c|}
\hline CSF & Tube 1 & Tube 4 & $\begin{array}{l}\text { Reference } \\
\text { range }\end{array}$ \\
\hline Appearance & No xanthochromia & No xanthochromia & $\begin{array}{l}\text { No } \\
\text { xanthochromia }\end{array}$ \\
\hline RBCs & 122 & 9 & None cells/uL \\
\hline WBCs & 59 & 50 & $<6$ cells/uL \\
\hline $\begin{array}{l}\text { Differential } \\
\text { (\%) }\end{array}$ & $\begin{array}{l}88 \% \text { lymphocytes, } 3 \% \text { PMNs, } 9 \% \\
\text { monocytes }\end{array}$ & $\begin{array}{l}74 \% \text { lymphocytes, } 24 \% \text { monocytes, } 1 \% \text { PMNs, } 1 \% \\
\text { basophils }\end{array}$ & \\
\hline Protein & 98.9 & & $15-45 \mathrm{mg} / \mathrm{dL}$ \\
\hline Glucose & 80 & & $40-70 \mathrm{mg} / \mathrm{dL}$ \\
\hline
\end{tabular}

\section{TABLE 1: Cerebrospinal fluid profile.}

CSF profile on day of presentation, suggestive of viral meningitis.

CSF: Cerebrospinal fluid; PMNs: Polymorphonuclear cells.

\begin{tabular}{|c|c|c|}
\hline Test & Reference range & Result \\
\hline Lactate dehydrogenase (serum) & 122-241 U/L & 237 \\
\hline C-reactive protein (serum) & $0.0-1.0 \mathrm{mg} / \mathrm{dL}$ & 0.4 \\
\hline HIV 1,2 Ab (serum) & Negative & Negative \\
\hline HIV p24 Ag (serum) & Not detected & Not detected \\
\hline Lyme Ab with Western Blot reflex (serum) & $<0.90 \mathrm{LI}$ & 0.18 \\
\hline Anaplasma PCR (serum) & Negative & Negative \\
\hline Babesia PCR (serum) & Negative & Negative \\
\hline Babesia smear (serum) & Negative & Negative \\
\hline Enterovirus PCR (CSF) & Not detected & Not detected \\
\hline Adenovirus PCR (serum) & Not detected & Not detected \\
\hline $\begin{array}{l}\text { Direct fluorescent Ab PCR including influenza A and B, metapneumovirus, rhinovirus, } \\
\text { parainfluenza virus, respiratory syncytial virus, Chlamydia pneumoniae, and Mycoplasma } \\
\text { (nasal swab) }\end{array}$ & Negative & Negative \\
\hline Influenza H1N1 (2009) PCR (serum) & Negative & Negative \\
\hline West Nile IgG (CSF) & Negative & Negative \\
\hline Herpes simplex virus 1,2 PCR (CSF) & Not detected & Not detected \\
\hline CSF culture & Negative & Negative \\
\hline Cryptococcal Ag (CSF) & Not detected & Not detected \\
\hline Cytomegalovirus PCR (CSF) & Not detected & Not detected \\
\hline Leptospira Ab (serum) & Negative & Negative \\
\hline Hepatitis general panel (Hepatitis B surface $A g, A b, C$ Ab with PCR reflex, A Ab) (serum) & Negative & Negative \\
\hline ANA by IFA with reflex (serum) & $<1: 80$ & $<1: 80$ \\
\hline ANCA screen with reflex (serum) & Negative & Negative \\
\hline dsDNA with reflex (serum) & $<10 \mathrm{IU} / \mathrm{mL}$ & 1.7 \\
\hline
\end{tabular}




\section{Cureus}

QuantiFERON-TB (serum)

Negative Negative

Quantitative buffy coat for blood parasites screen (serum)

Negative for intra- Negative for intraerythrocyte erythrocyte

Serotonin (serum) parasites parasites

Rheumatoid factor (serum)

$56-244 \mathrm{ng} / \mathrm{mL} \quad 25$

$<14 \mathrm{IU} / \mathrm{mL} \quad<10$

SS-A (serum)

$<7.0 \mathrm{U} / \mathrm{mL}$

2.6

SS-B (serum)

$<7.0 \mathrm{U} / \mathrm{mL}$

$<0.3$

Autoimmune encephalopathy panel (serum and CSF)

Negative

Negative

St. Louis encephalitis IgM (CSF)

Negative Negative

Eastern equine encephalitis IgM (CSF)

Negative

Negative

Western equine encephalitis IgM (CSF)

Negative

Negative

Powassan IgM sent to CDC (serum)

Negative

\section{TABLE 2: Serum and cerebrospinal fluid tests.}

Serum and CSF tests were negative for many potential causes of meningoencephalitis but serum IgM eventually returned positive for Powassan virus. The SS-A serology test was likely either a false positive or consistent with the patient's history of reactive arthritis.

Ab: Antibody; Ag: Antigen, PCR: Polymerase chain reaction; IgG: Immunoglobulin G; ANA: Anti-nuclear antibody; IFA: Indirect fluorescent antibody; ANCA: Antineutrophil cytoplasmic antibodies; dsDNA: Double-stranded DNA; IgM: Immunoglobulin M.

Empiric antibiotics (vancomycin and ceftriaxone) and antiviral (acyclovir) were started for possible meningitis or encephalitis. CT scans of the chest, abdomen, and pelvis were performed due to a history of CLL, which showed no evidence of bulky lymphadenopathy. A peripheral blood smear was consistent with chronic CLL. MRI of the brain with and without contrast showed mild generalized cerebral and cerebellar atrophy and a few small nonspecific T2 fluid-attenuated inversion recovery (FLAIR) hyperintensities with no enhancement on T1-weighted MRI (Figure 1). An overnight continuous EEG did not reveal any seizures. The patient's mental status deteriorated over the hospital day 1-2 and he developed profound expressive aphasia and ataxia. He required a non-rebreather to maintain oxygen saturation above $90 \%$ because of failure to protect his airway. He was transferred to the ICU and intubated for airway protection on hospital day 6. Given that his CSF bacterial cultures showed no growth to this point, and herpes simplex virus (HSV) and varicella-zoster virus (VZV) polymerase chain reactions (PCRs) were negative, empiric antibiotics and acyclovir were discontinued and he was given a five-day course of IV methylprednisolone with gradual improvement in neurologic status. MRI brain with gadolinium was repeated and showed extensive high signal with mild restricted diffusion involving bilateral cerebellar hemispheres, consistent with cerebellitis (Figure 2).

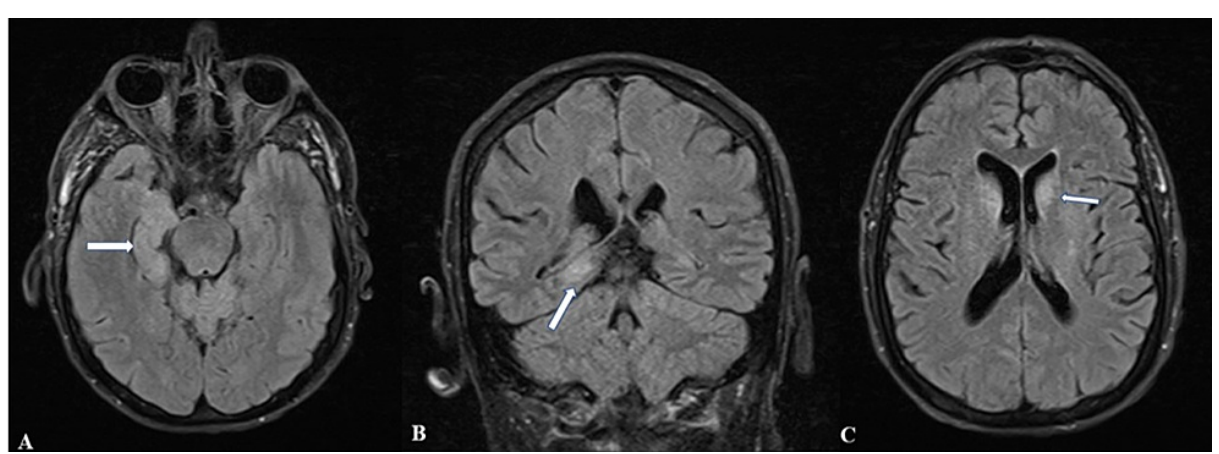

FIGURE 1: Initial MRI of the brain.

MRI of the brain showing FLAIR hyperintensities in the right temporal lobe (panel A and B) as well as in the bilateral caudate nucleus (panel C).

FLAIR: Fluid-attenuated inversion recovery. 


\section{Cureus}

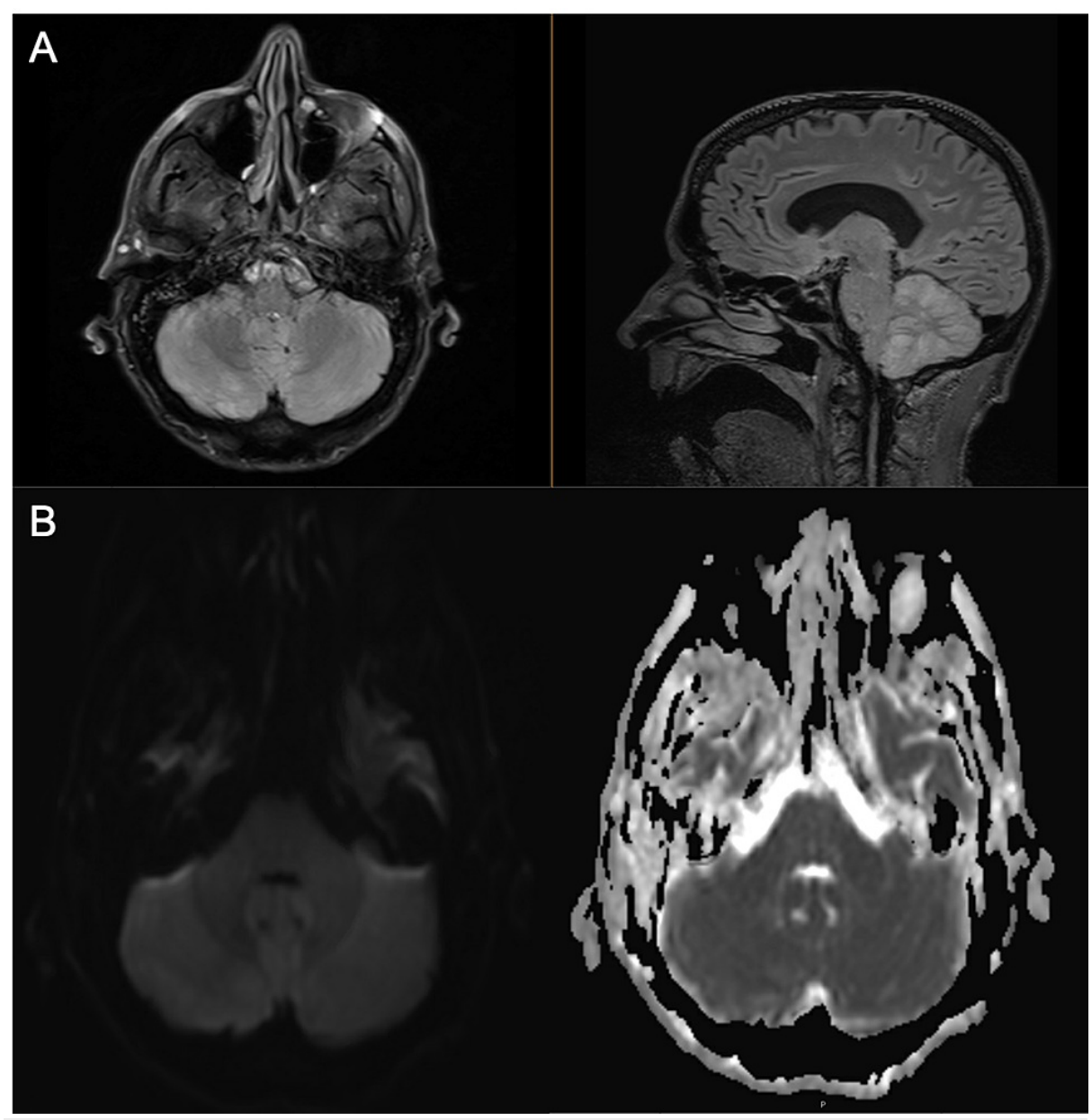

\section{FIGURE 2: Follow-up MRI of the brain.}

A) MRI of the brain showing evidence of cerebellar FLAIR enhancement, consistent with cerebellitis B) DWI image with corresponding ADC map showing mild restricted diffusion.

ADC: Apparent diffusion coefficient; DWI: Diffusion-weighted imaging; FLAIR: Fluid-attenuated inversion recovery.

Subsequently, CSF serologies returned positive for POWV, which supported a diagnosis of Powassan meningoencephalitis. The infectious diseases service was consulted and recommended no further treatment in the in-patient setting. After hospital day 34, the patient was transferred to a skilled nursing facility and underwent physical and occupational therapy.

Upon evaluation in the out-patient clinic a few weeks later, the patient demonstrated significant left-sided ataxia and expressive aphasia. On subsequent three-month follow-up, he had developed spasticity of both upper extremities, resulting in difficulty using a keyboard and occasional pain in the left upper extremity. On this examination, he was noted to have increased tone of the left upper extremity and flexed posture of biceps and fingers. Speech therapy was recommended and physical and occupational therapy was continued. He was prescribed gabapentin $300 \mathrm{mg}$ three times daily (TID) and baclofen $10 \mathrm{mg}$ TID, and referred for botulinum toxin (Botox) injections. A year after his initial admission, the patient underwent a follow-up MRI which showed progressive cerebellar atrophy compared to previous imaging with full resolution of hyperintensities (Figure 3). 


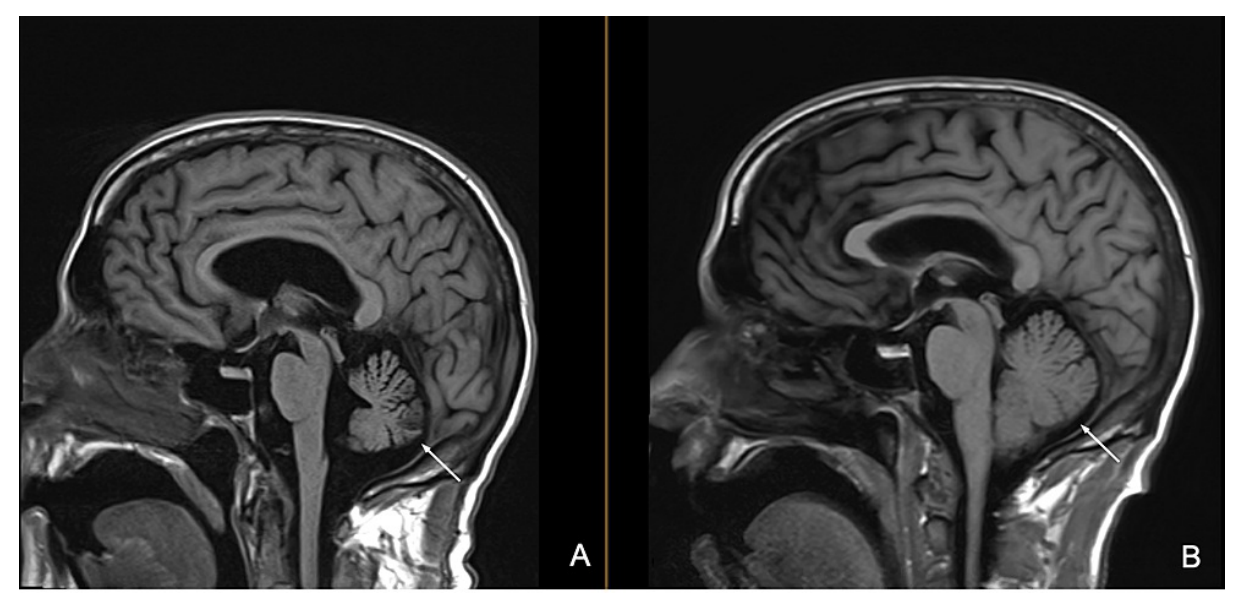

FIGURE 3: One-year follow-up MRI of the brain.

Follow-up MRI one year after initial presentation showing progressive cerebellar atrophy (A) compared to previous imaging $(\mathrm{B})$ with an improvement of hyperintensities.

Tone improved in both upper extremities with baclofen and Botox injections, and his wife noted he was not complaining of pain on this regimen. However, the patient continued to have flexed posture of the 4 th and 5th digits of both hands and developed a head drop with decreased neck extensor tone. Botox regimen was adjusted to include these muscle groups with partial effect. To date, the patient has continued with Botox injections every three months and underwent a procedure to receive a baclofen pump for refractory bilateral upper extremity spasticity with subsequent improvement in his stiffness and tone. He is wheelchair-bound and continues to have ataxia and expressive aphasia but is alert and oriented and able to follow commands.

\section{Discussion}

The extent of neurologic involvement seen in POWV infection varies widely. Neurologic symptoms seen in POWV include altered mental status, seizures, headache, memory impairment, blurry vision, diplopia, nystagmus, upward gaze palsy, dizziness, spastic and flaccid paralysis, and positive Babinski sign $[5,7]$. Upper and lower motor neuron involvement is more significant in POWV infection than in other causes of viral encephalitis [5]. Additionally, there is some evidence that POWV may be similar to hemorrhagic viruses such as dengue and yellow fever, as some cases of POWV show intraparenchymal hemorrhage and subdural hematoma [5]. Thus, POWV may also present with focal deficits such as hemiplegia and hemiparesis in the setting of intracranial bleeding. Neuropsychiatric symptoms such as anhedonia and depression may also be seen. Long-term sequelae are variable, ranging from severe, disabling residual deficits to a return to nearbaseline function with a normal neurologic exam [8]. As noted earlier, our patient developed expressive aphasia and spasticity of both upper extremities for which he underwent physical, occupational, and speech therapy as well as treatment with an oral muscle relaxant and Botox injections. Apart from neurologic symptoms, respiratory failure may also occur from depressed consciousness leading to failure to protect the airway or inadequate oxygenation. Intubation may be needed, as demonstrated by our case [9].

Radiologic findings in POWV meningoencephalitis also vary widely. In the acute setting, a CT scan is unlikely to show abnormalities unless there is intracranial bleeding [5]. MRI is more likely to show ischemia on T2 FLAIR but these are not universal. Hyperintensity is most often seen in the periventricular white matter, perivascular space, and deep white matter. These abnormalities tend to improve once symptoms have resolved [5, 8]. Abnormal contrast enhancement is not typically seen, though subtle enhancement of the vermis and diffuse cerebellar parenchymal and leptomeningeal enhancement have been reported in some cases [8]. The progression of MRI findings over time with simultaneous gradual improvement in our patient's neurologic status agrees with imaging findings of POWV neuroinvasive disease reported in the literature. T2 FLAIR hyperintensities were seen and subsequently resolved on repeat imaging concurrently with improvements in the patient's neurologic status. While initial imaging revealed hyperintensities involving the bilateral hippocampal/parahippocampal gyrus and caudate heads, subsequent scans showed resolution of these findings with a new extensive high signal with mild restricted diffusion involving bilateral cerebellar hemispheres.

Our patient's history of CLL is an interesting confounder in this case. Immunosuppression secondary to CLL leads to an increased risk of infections, even for patients not undergoing immunosuppressive treatment. This may be in part due to the impaired T-cell immune response and hypogammaglobulinemia known to accompany CLL [10]. Thus, patients with CLL, even those like our patient who is not on immunosuppressive medications, are prone to new infections or reactivations of viral infections. This underscores the importance of a higher index of suspicion for neuroinvasive infection in all immunosuppressed patients. 
Although infection with POWV is rare, the potential for rapid clinical deterioration and the need for urgent supportive care warrant consideration of POWV when tick-borne illness is suspected, particularly in the Northeastern United States and Great Lakes regions. As demonstrated by our case, POWV can cause severe meningitis and encephalitis with serious long-term neurologic sequelae. Though our patient survived, POWV infection can be fatal in up to $20 \%$ of cases, underscoring the importance of early supportive care. Physicians should be prepared to recognize the common presenting symptoms of POWV, including fever, headache, confusion, encephalopathy, and neurological symptoms, and to provide both aggressive supportive care in the initial phases and appropriate management of long-term neurologic sequelae. In many cases, multidisciplinary care with occupational, physical, and/or speech therapy may be helpful. Patient education is also important, as no vaccination and specific treatment exist at this time, and tick bite prevention remains the best defense against POWV infection.

\section{Conclusions}

It is important to have a broad differential diagnosis when evaluating patients presenting with symptoms of neuroinvasive infection. While rare, as demonstrated by our patient's case, infection with POWV may lead to rapid clinical deterioration and long-term neurologic deficits. Our patient exhibited ataxia, expressive aphasia, and spasticity as consequences of severe POWV infection. Immunosuppression may play a role in a patient's susceptibility to neuroinvasion with POWV. A thorough history, clinical examination, and diagnostic testing are necessary to guide early supportive care. Appropriate outpatient rehabilitation and management of sequelae can minimize long-term morbidity. Patient education remains the best preventive strategy.

\section{Additional Information \\ Disclosures}

Human subjects: Consent was obtained or waived by all participants in this study. Conflicts of interest: In compliance with the ICMJE uniform disclosure form, all authors declare the following: Payment/services info: All authors have declared that no financial support was received from any organization for the submitted work. Financial relationships: All authors have declared that they have no financial relationships at present or within the previous three years with any organizations that might have an interest in the submitted work. Other relationships: All authors have declared that there are no other relationships or activities that could appear to have influenced the submitted work.

\section{References}

1. Colman D, Peaslee S: Powassan virus in a hunter returning from a trip in the Adirondack Park . Wilderness Environ Med. 2020, 31:87-90. 10.1016/j.wem.2019.09.002

2. CDC Powassan Virus Statistics \& Maps . (2020). Accessed: March 28, 2021: https://www.cdc.gov/powassan/statistics.html.

3. Kemenesi G, Bányai K: Tick-borne flaviviruses, with a focus on Powassan virus . Clin Microbiol Rev. 2019, 32:10.1128/CMR.00106-17

4. Hermance ME, Thangamani S: Powassan virus: an emerging arbovirus of public health concern in North America. Vector Borne Zoonotic Dis. 2017, 17:453-462. 10.1089/vbz.2017.2110

5. Fatmi SS, Zehra R, Carpenter DO: Powassan virus-a new reemerging tick-borne disease . Front Public Health. 2017, 5:342. 10.3389/fpubh.2017.00342

6. Corrin T, Greig J, Harding S, Young I, Mascarenhas M, Waddell LA: Powassan virus, a scoping review of the global evidence. Zoonoses Public Health. 2018, 65:595-624. 10.1111/zph.12485

7. Gholam BI, Puksa S, Provias JP: Powassan encephalitis: a case report with neuropathology and literature review. CMAJ. 1999, 161:1419-1422.

8. Piantadosi A, Rubin DB, McQuillen DP, et al.: Emerging cases of Powassan virus encephalitis in New England: clinical presentation, imaging, and review of the literature. Clin Infect Dis. 2016, 62:707-713. 10.1093/cid/civ1005

9. Davis LE, Beckham JD, Tyler KL: North American encephalitic arboviruses. Neurol Clin. 2008, 26:727-757. 10.1016/j.ncl.2008.03.012

10. Melchardt T, Weiss L, Greil R, Egle A: Viral infections and their management in patients with chronic lymphocytic leukemia. Leuk Lymphoma. 2013, 54:1602-1613. 10.3109/10428194.2012.755178 